\title{
QUALIDADE DE VIDA DE PACIENTES COM BRONQUIECTASIA EM ACOMPANHAMENTO AMBULATORIAL
}

\section{Quality of life of patients with bronchiectasis following outpatient treatment \\ Calidad de vida de pacientes con Bronquiectasia en seguimiento en el ambulatorio}

\section{Theila Oliveira Simas}

Faculdade Santa Terezinha - CEST - São Luís (MA) - Brasil

\section{Carlos Martins Neto}

Hospital Universitário da Universidade Federal do Maranhão - HU/UFMA - São Luís (MA) - Brasil

\section{Olga Lorena Maluf Guará Beserra}

Faculdade Santa Terezinha - CEST - São Luís (MA) - Brasil

\section{Emerson Silva Brito}

Faculdade Santa Terezinha - CEST - São Luís (MA) - Brasil

\section{João Victor Mota}

Faculdade Santa Terezinha - CEST - São Luís (MA) - Brasil

\author{
Maysa Ferraz Reis Barroso \\ Hospital Universitário da Universidade Federal do Maranhão - HU/UFMA - São Luís (MA) - Brasil
}

\section{RESUMO}

Objetivo: Avaliar a qualidade de vida dos pacientes com bronquiectasia em acompanhamento ambulatorial. Métodos: Estudo transversal, quantitativo e descritivo, realizado com 31 pacientes com diagnóstico de bronquiectasia, desenvolvido no setor de fisioterapia respiratória da Clínica-escola Santa Edwiges - APAE e no Ambulatório de Pneumologia do Programa de Assistência ao Paciente Asmático do Hospital Universitário (PAPA) em São Luís, Maranhão, de julho a agosto de 2015, em que se aplicaram um questionário socioeconômico e demográfico e o questionário Short-Form-36 (SF-36) para avaliação da qualidade de vida. Realizou-se análise descritiva dos dados no software Epi Info, versão 7.0, sendo apresentados na forma de tabelas, através de valores absolutos (n), relativos (\%), média, mediana, valor mínimo, valor máximo e desvio padrão. Resultados: Observou-se idade média de 46,96 $\pm 15,52$ anos, predomínio do sexo feminino $(54,8 \%$; $\mathrm{n}=17)$, cor parda $(58,0 \% ; \mathrm{n}=18)$, casados $(35,4 \% ; \mathrm{n}=11)$ e ensino fundamental completo $(51,6 \% ; \mathrm{n}=16)$. Quanto à qualidade de vida, os domínios aspectos emocionais $(16,1 \pm 30,9)$, aspectos físicos $(19,3 \pm 30,7)$, estado geral de saúde $(35,8 \pm 19,5)$ e capacidade funcional $(50,3 \pm 25,0)$ apresentaramse comprometidos, enquanto os domínios vitalidade $(55,0 \pm 27,4)$, aspectos sociais $(60,2 \pm 24,9)$, saúde mental $(66,7 \pm 23,6)$ e dor $(68,0 \pm 27,7)$ apresentaram bons resultados. Conclusão: A avaliação do SF-36 apontou uma redução da qualidade de vida em pacientes com bronquiectasia nos seguintes domínios: aspectos emocionais, aspectos físicos, estado geral de saúde e capacidade funcional.

Descritores: Pneumopatias; Bronquiectasia; Qualidade de Vida.

\section{ABSTRACT}

Objective: To assess the quality of life of patients with bronchiectasis following outpatient treatment. Methods: Quantitative descriptive crosssectional study of 31 patients diagnosed with bronchiectasis carried out in the Department of Respiratory Physical Therapy of the Santa Edwiges Teaching Clinic (Clinica-escola Santa Edwiges - APAE) and in the Outpatient Pulmonology Clinic of the Asthma Patient Care Program of the University Hospital (Programa de Assistência ao Paciente Asmático do Hospital Universitário - PAPA) in São Luís, Maranhão, from July to August 2015, using a socioeconomic and demographic questionnaire and the SF-36 questionnaire to assess quality of life. A descriptive analysis of the data was carried out in Epi Info, version 7.0 and the data are presented in tables using absolute (n) and relative (\%) values, mean, median, minimum value, maximum value and standard deviation. Results: The mean age of the participants was $46.96 \pm 15.52$ years and there was a predominance of women $(54.8 \%, n=17)$, pardos $(58.0 \%, n=18)$, married individuals $(35.4 \%, n=11)$ and complete elementary education (51.6\%, $n=16)$. Regarding quality of life, there was impairment of emotional aspects (16.1 \pm 30.9$)$, physical aspects (19.3 \pm 30.7$)$, general health status (35.8 \pm 19.5$)$ and functional capacity (50.3 \pm 25.0$)$, and vitality (55.0 \pm 27.4$)$, social aspects (60.2 \pm 24.9$)$, mental health (66.7 \pm 23.6$)$ and pain (68.0 27.7$)$ exhibited good results. Conclusion: The evaluation of the SF-36 indicated a decreased quality of life in patients with bronchiectasis in the following domains: emotional aspects, physical aspects, general health status and functional capacity.

Descriptors: Lung Diseases; Bronchiectasis; Quality of Life. 


\section{RESUMEN}

Objetivo: Evaluar la calidad de vida de los pacientes con Bronquiectasia en seguimiento en el ambulatorio. Métodos: Estudio transversal, cuantitativo y descriptivo realizado con 31 pacientes con diagnóstico de bronquiectasia desarrollado en el sector de fisioterapia respiratoria de la Clínica-escuela Santa Edwiges - APAE y en el Ambulatorio de Neumología del Programa de Asistencia al Paciente Asmático del Hospital Universitario (PAPA) de São Luís, Maranhão, entre julio y agosto de 2015 en el cual se aplicó un cuestionario socioeconómico y demográfico y el cuestionario Short-Form-36 (SF-36) para la evaluación de la calidad de vida. Se realizó un análisis descriptivo de los datos en el software Epi Info versión 7.0 que fueron presentados en tablas a través de los valores absolutos (n), los relativos (\%), la media, la mediana, el valor mínimo y máximo y la desviación típica. Resultados: Se observó la media de edad de 46,96士15,52 años, predominio del sexo femenino $(54,8 \% ; n=17)$, el color pardo $(58,0 \% ; n=18)$, estado civil casado(a) $(35,4 \%$; $n=11)$ y con educación primaria completa $(51,6 \% ; n=16)$. Respecto la calidad de vida, los dominios aspectos emocionales $(16,1 \pm 30,9)$, aspectos físicos $(19,3 \pm 30,7)$, estado general de salud $(35,8 \pm 19,5)$ y capacidad funcional $(50,3 \pm 25,0)$ se presentaron comprometidos mientras los dominios vitalidad $(55,0 \pm 27,4)$, aspectos sociales $(60,2 \pm 24,9)$, salud mental $(66,7 \pm 23,6)$ y dolor $(68,0 \pm 27,7)$ presentaron buenos resultados. Conclusión: La evaluación del SF-36 apuntó una reducción de la calidad de vida de pacientes con bronquiectasia para los siguientes dominios: aspectos emocionales, aspectos fisicos, estado general de salud y capacidad funcional.

Descriptores: Enfermedades Pulmonares; Bronquiectasia; Calidad de Vida.

\section{INTRODUÇÃO}

A bronquiectasia caracteriza-se por uma dilatação anormal e irreversível dos brônquios, com destruição das paredes das vias aéreas, que ocorre por meio de infecções e inflamações recorrentes causadas por infecções respiratórias graves ou repetidas, resultando em prejuízo do clearance, acúmulo de secreção nas áreas afetadas e colonização bacteriana. É mais prevalente em mulheres de meia-idade, tendo como principais manifestações clínicas: tosse crônica, comumente associada à expectoração purulenta de odor fétido, predominantemente pela manhã; dispneia; hemoptise; febre; fadiga e perda de peso ${ }^{(1)}$.

Nos países desenvolvidos, principalmente, os avanços no tratamento com antibióticos, nos últimos anos, proporcionaram uma diminuição da bronquiectasia de origem infecciosa, porém, nos países em desenvolvimento, a bronquiectasia pós-infecciosa ainda consiste em um importante problema de saúde pública e sua alta morbidade interfere de forma significativa na qualidade de vida dos seus portadores ${ }^{(2)}$.

O aumento da expectativa de vida decorrente do desenvolvimento tecnológico e científico ocorrido nas últimas décadas teve amplo impacto na prevalência de doenças crônicas, como a bronquiectasia. Logo, o interesse pelo conceito de qualidade de vida em relação à saúde tornou-se de grande importância para a sociedade, pois é um indicador de saúde pública cada vez mais utilizado para controlar o impacto da doença em uma população(3).

Entende-se que a qualidade de vida se relaciona a um amplo espectro de condições internas e externas ao indivíduo. As condições externas predispõem uma vida de qualidade, sem, no entanto, serem os fatores determinantes. A qualidade de vida é determinada pelo próprio indivíduo, incluindo uma experiência interna de satisfação e bem-estar com o seu processo de viver. Assim, mesmo com todas as condições externas favoráveis, pode haver redução da qualidade de vida. No entanto, seria improvável o indivíduo apresentar uma boa qualidade de vida sem uma dessas condições ${ }^{(4)}$.

Vários são os estudos sobre doenças pulmonares crônicas, porém, a respeito da bronquiectasia e qualidade de vida, ainda há uma escassez. As doenças pulmonares, incluindo a bronquiectasia, representam um problema de saúde pública não só em países subdesenvolvidos e emergentes, mas também em países industrializados, em função da sua prevalência e do seu impacto econômico, com altos custos com internações hospitalares e medicamentos, tanto para o Estado quanto para os pacientes ${ }^{(5)}$. Tendo isso em vista, a presente pesquisa teve como objetivo avaliar a qualidade de vida dos pacientes com bronquiectasia em acompanhamento ambulatorial.

\section{MÉTODOS}

Trata-se de um estudo quantitativo, descritivo, de caráter transversal, realizado no período de julho a agosto de 2015, tendo sido desenvolvido no setor de fisioterapia respiratória da Clínica-escola Santa Edwiges - APAE e no Ambulatório de Pneumologia do Programa de Assistência ao Paciente Asmático do Hospital Universitário em São Luís, Maranhão, Brasil.

Esses locais são ambulatórios de referência, os quais possuem pacientes com diversas patologias respiratórias, incluindo a bronquiectasia. Foram selecionados para este estudo, de forma não probabilística e por conveniência, 31 pacientes. Como critérios de inclusão tinham-se pacientes de ambos os sexos, com idade igual ou superior a 18 anos, e diagnóstico confirmado de bronquiectasia. Como critérios de exclusão, tinham-se aqueles que exacerbaram e não puderam estar presentes no momento da avaliação, e que não possuíam capacidade cognitiva para compreender e responder aos questionários. 
Os dados foram coletados nos ambulatórios, em uma sala reservada, em momento antes ou após o atendimento fisioterapêutico. Os pacientes eram convidados individualmente pelos pesquisadores, informados sobre os objetivos do estudo e como deveriam realizar o preenchimento do questionário. Então, após esse momento, aqueles que aceitaram participar do estudo assinaram o Termo de Consentimento Livre e Esclarecido.

Iniciou-se a coleta de dados por meio da aplicação de dois instrumentos: um questionário socioeconômico e demográfico, e um questionário de avaliação da qualidade de vida. O primeiro questionário, elaborado pelos pesquisadores, continha questões como sexo, idade, cor referida da pele, escolaridade, local onde mora, renda mensal, entre outras.

Na sequência, aplicou-se o questionário Short-Form-36, versão do Medical Outcomes Study (SF-36), validado para o Brasil, largamente utilizado na avaliação da Qualidade de vida (QV). Ele tem 36 itens, subdivididos em 8 domínios, que abordam a capacidade funcional, aspectos físicos, dor, estado geral de saúde, vitalidade, aspectos sociais, aspectos emocionais e saúde mental $^{(6)}$. O participante recebe um escore em cada domínio, que varia de 0 a 100, sendo 0 o pior e 100 o melhor ${ }^{(7)}$.

Para análise de dados, utilizou-se estatística descritiva com auxílio do software Epi info, versão 7.0, sendo apresentados na forma de tabelas, através de valores absolutos (n), relativos (\%), média, mediana, valor mínimo, valor máximo e desvio padrão (DP).

A pesquisa foi aprovada pelo Comitê de Ética em Pesquisa sob Parecer n ${ }^{\circ} 1.053 .250$, respeitando a Resolução $n^{\circ} 466 / 2012$ do Conselho Nacional de Saúde.

\section{RESULTADOS}

Fizeram parte do estudo 31 pacientes portadores de bronquiectasia. Houve um predomínio do sexo feminino (54,8\%; $\mathrm{n}=17$ ), com faixa etária entre 18 e 74 anos, com média de 46,96 $\pm 15,52$ anos. A maioria encontrava-se com idade $\geq 54$ anos $(35,4 \% ; n=11)$.

Quanto à cor referida da pele, houve maior frequência da cor parda $(58,0 \% ; \mathrm{n}=18)$, oriundos do interior do estado do Maranhão $(65,4 \% ; n=20)$, casados $(35,4 \% ; n=11)$, com ensino fundamental completo $(51,6 \% ; n=16)$. A maioria possuía renda mensal de 1 (um) salário mínimo $(61,3 \% ; n=19)$ e morava em casa própria $(86,6 \% ; n=26)$, com predomínio daqueles que utilizam transporte coletivo $(70,9 \% ; n=22)$ como principal meio de locomoção. A amostra não possui plano de saúde privado (100\%; $\mathrm{n}=31)($ Tabela I).

Tabela I - Perfil socioeconômico e demográfico dos pacientes com bronquiectasia em acompanhamento ambulatorial. São Luís, Maranhão, Brasil, 2015.

\begin{tabular}{|c|c|c|}
\hline Variável & $\mathbf{n}$ & $\%$ \\
\hline \multicolumn{3}{|l|}{ Sexo } \\
\hline Masculino & 14 & 45,1 \\
\hline Feminino & 17 & 54,9 \\
\hline \multicolumn{3}{|l|}{ Faixa etária } \\
\hline $18-29$ & 6 & 19,3 \\
\hline $30-42$ & 4 & 12,9 \\
\hline $43-54$ & 10 & 32,3 \\
\hline$>54$ & 11 & 35,5 \\
\hline \multicolumn{3}{|l|}{ Cor referida da pele } \\
\hline Branca & 5 & 16,1 \\
\hline Preta & 8 & 25,9 \\
\hline Parda & 18 & 58,0 \\
\hline \multicolumn{3}{|l|}{ Local de origem } \\
\hline São Luís & 11 & 35 \\
\hline Interior do estado & 20 & 65 \\
\hline \multicolumn{3}{|l|}{ Escolaridade } \\
\hline Analfabeto & 1 & 3,3 \\
\hline Ensino fundamental completo & 16 & 51,6 \\
\hline Ensino médio completo & 13 & 41,9 \\
\hline Superior completo & 1 & 3,2 \\
\hline \multicolumn{3}{|l|}{ Estado Civil } \\
\hline Casado & 11 & 35,5 \\
\hline União estável & 6 & 19,4 \\
\hline Divorciado & 5 & 16,2 \\
\hline Solteiro & 7 & 22,5 \\
\hline Viúvo & 2 & 6,4 \\
\hline
\end{tabular}




\begin{tabular}{lcc}
\hline Renda Mensal & 6 & \\
Nenhuma & 19 & 19,3 \\
1 salário mínimo & 0 & 61,4 \\
2 salários mínimos & 6 & 0 \\
3 salários mínimos & 0 & 19,3 \\
> 3 salários mínimos & & 0 \\
Casa onde mora & 26 & 86,6 \\
$\quad$ Própria & 3 & 9,6 \\
Alugada & 2 & 4 \\
Cedida & 0 & 00 \\
Meio de transporte & 22 & 70,9 \\
Carro próprio & 9 & 29,1 \\
Transporte coletivo & 0 & 0 \\
Outros & 31 & 100 \\
Plano de saúde privado & & \\
Sim & & \\
Não &
\end{tabular}

Legenda: n: número absoluto de participantes; \%: número relativo.

Quando avaliado o SF-36, observaram-se menores escores, ou seja, uma QV ruim, nos domínios relacionados aos aspectos emocionais $(16,1 \pm 30,9)$, aspectos físicos $(19,3 \pm 30,7)$, estado geral de saúde $(35,8 \pm 19,5)$ e capacidade funcional $(50,3 \pm 25,0)$. Observaram-se maiores escores, ou seja, QV boa, nos domínios vitalidade $(55,0 \pm 27,4)$, aspectos sociais $(60,2 \pm 24,9)$, saúde mental $(66,7 \pm 23,6)$ e dor $(68,0 \pm 27,7)$.

Tabela II - Escore geral do SF-36 avaliado em uma amostra de pacientes com bronquiectasia em acompanhamento ambulatorial. São Luís, Maranhão, Brasil, 2015.

\begin{tabular}{lccccc}
\hline Domínios & n & Média & DP & Mediana & Mínimo - Máximo \\
\hline Capacidade funcional & 31 & 50,3 & 25,0 & 50 & $0-100$ \\
Aspectos físicos & 31 & 19,3 & 30,7 & 0 & $0-100$ \\
Dor & 31 & 68,0 & 27,7 & 62 & $22-100$ \\
Estado geral de saúde & 31 & 35,8 & 19,5 & 32 & $5-75$ \\
Vitalidade & 31 & 55,0 & 27,4 & 60 & $15-100$ \\
Aspectos sociais & 31 & 60,2 & 24,9 & 63,0 & $10-100$ \\
Aspectos emocionais & 31 & 16,1 & 30,9 & 0 & $0-100$ \\
Saúde mental & 31 & 66,7 & 23,6 & 64,0 & $0-100$ \\
\hline
\end{tabular}

Legenda: DP: Desvio Padrão; n: número absoluto de participantes.

\section{DISCUSSÃO}

A avaliação da qualidade de vida no grupo de pacientes com bronquiectasia demonstrou que os domínios aspectos emocionais, aspectos físicos, estado geral de saúde e capacidade funcional tiveram os piores resultados, ou seja, demonstraram maior grau de comprometimento da QV, enquanto os domínios vitalidade, aspectos sociais, saúde mental e dor obtiveram resultados melhores, demonstrando que a QV, do ponto de vista desses aspectos, não se encontra comprometida.

Assim como os resultados da atual pesquisa, a literatura aponta que a bronquiectasia é uma doença pulmonar infecciosa crônica que acomete, preferencialmente, o sexo feminino ${ }^{(8-10)}$. Há evidências que o hipoestrogenismo, observado em mulheres após a menopausa, atenua a resposta imune e predispõe o organismo à invasão microbiana e infecção que, quando atinge repetidas vezes o sistema respiratório, pode acarretar ou comprometer ainda mais o quadro da paciente ${ }^{(11)}$.

Similar ao observado na presente pesquisa, a idade média de pessoas com bronquiectasia em outros estudos varia de 28,7 a 48,0 anos, até 55 anos, período de maior produtividade de suas vidas ${ }^{(9,12)}$. Em recente estudo sul-coreano foi demonstrada a prevalência de bronquiectasia em $9,1 \%$ das pessoas com idade entre 23 e 86 anos. A prevalência foi maior em mulheres que em homens $(11,5$ versus $7,9 \%)$ e aumentou proporcionalmente com a idade ${ }^{(10)}$.

Resultado divergente da atual pesquisa foi encontrado em estudo realizado no estado de São Paulo, com pacientes com bronquiectasia de etiologia tuberculosa e não tuberculosa, em que o maior número de bronquiectasias ocorreu em indivíduos da cor branca e negra ( $41 \%$ e $39 \%$, respectivamente) e apenas $20 \%$ da cor parda ${ }^{(13)}$. Este fato pode ser explicado pelo alto índice 
de miscigenação no Brasil. Segundo $\operatorname{IBGE}^{(14)}$, em 2014, mais da metade (53,6\%) dos brasileiros se declaravam como de cor ou raça preta ou parda.

A associação entre raça e saúde é bastante concreta, confirmada por pesquisa em nível nacional que atesta desigualdades sofridas pelos negros no que diz respeito à saúde. A prevalência de doenças crônicas autorreferidas foi significativamente maior nas pessoas negras quando comparadas às brancas ${ }^{(15)}$.

O presente estudo mostrou grande número de pacientes com baixa escolaridade e habitação em locais de pouca infraestrutura, o que pode refletir em baixas condições socioeconômicas. A escolaridade de pacientes com bronquiectasia se apresenta baixa em outros estudos ${ }^{(4,16)}$, que é algo característico de países em desenvolvimento ${ }^{(17)}$. A educação inadequada, juntamente com a habitação precária, a dificuldade ao acesso médico e desnutrição, aumentam a prevalência e a morbidade das bronquiectasias ${ }^{(18)}$. A gestão da doença, até então negligenciada, que atinge proporções quase epidêmicas em alguns grupos étnicos e é uma fonte contínua de considerável morbidade e mortalidade, requer uma abordagem abrangente e multidisciplinar ${ }^{(18)}$.

Em relação à avaliação da qualidade de vida, observaram-se escores baixos no estudo em questão nos seguintes domínios: aspectos emocionais, aspectos físicos, estado geral de saúde e capacidade funcional. Resultados semelhantes foram encontrados em pesquisa que avaliou a qualidade de vida de idosos, em sua maioria fumantes ou ex-fumantes, utilizando o SF-36, em que os piores escores foram observados nos domínios aspectos físicos $(49,2 \pm 40,9)$, dor $(54,8 \pm 22,9)$ e estado geral de saúde $(59,0 \pm 20)^{(7)}$.

Em outra pesquisa, com pacientes pneumopatas, utilizando o mesmo instrumento, observou-se que os domínios de qualidade de vida com maior comprometimento foram capacidade funcional, aspectos físicos, estado geral de saúde e vitalidade ${ }^{(19)}$. A perda da capacidade física e da qualidade de vida pode também estar relacionada com alterações psíquicas do paciente. As limitações fisiológicas e funcionais da habilidade física estão acompanhadas de fatores psicológicos, resultando em isolamento social e deterioração da qualidade de vida ${ }^{(20)}$.

Os maiores escores observados na atual pesquisa foram relacionados aos domínios vitalidade, aspectos sociais, saúde mental e dor. Resultados semelhantes foram observados em pesquisa que avaliou a qualidade de vida de pacientes com Doença Pulmonar Obstrutiva Crônica (DPOC) através do SF-36, em que alguns dos melhores escores foram relacionados aos domínios aspectos emocionais $(70,42 \pm 30,16)$, aspectos sociais $(70,00 \pm 20,44)$ e dor $(64,85 \pm 25,33)^{(21)}$.

Observou-se, nesta pesquisa, que o domínio aspectos emocionais é o menor entre todos os estudados. Isto se justifica na análise dos dados relacionados à qualidade de vida das pessoas com problemas respiratórios crônicos que integraram o grupo de convivência, em que ter qualidade de vida envolve a superação e o controle de sentimentos conflitantes que são trazidos pela doença respiratória crônica. Medo de crises respiratórias, vergonha, dificuldade na aceitação da doença e perda de prazeres foram manifestados pelos integrantes do estudo como interferindo em sua qualidade de vida, demonstrando insegurança em relação à possibilidade de controlar a doença, o que pode ameaçar seu futuro, especialmente quando percebem que o tratamento não é efetivo e quando não conseguem manter o controle da situação ${ }^{(22)}$.

Esses pacientes apresentam altos índices de ansiedade e depressão, bem como diminuição do desempenho cognitivo. Temse sugerido que as mudanças no humor resultam da autopercepção negativa e do prejuízo funcional, os quais se relacionam diretamente com a diminuição da capacidade física ${ }^{(23)}$, sendo este também um dos domínios pior avaliados na presente amostra. Isto mostra que a bronquiectasia é uma doença que compromete diversas áreas da vida do portador, afetando pessoas de várias idades e, em sua maioria, em idade economicamente ativa. Portanto, seu tratamento deve ser multidisciplinar e individualizado, incluindo cinesioterapia respiratória e uso de broncodilatadores e corticosteróides quando indicado para, dessa forma, melhorar a qualidade de vida desses indivíduos e evitar a progressão da doença ${ }^{(24)}$.

Mesmo não tendo sido analisado na presente pesquisa, torna-se necessário mencionar que o número de exacerbações da doença pode influenciar a qualidade do tratamento, uma vez que sua diminuição impacta positivamente na qualidade de vida. Finalmente, a avaliação do tratamento com base nos questionários de qualidade de vida parece ser uma das melhores formas de avaliar esses pacientes ${ }^{(25)}$.

A principal limitação encontrada na presente pesquisa foi relacionada à escassez de estudos que avaliem a qualidade de vida de pacientes com bronquiectasia. A maioria avalia pacientes com DPOC em geral. Portanto, são necessárias mais pesquisas com essa população, principalmente estudos com grupo-controle e com amostra maior, a fim de comparar a qualidade de vida desses pacientes com a população em geral e obter um parâmetro mais fidedigno de análise.

\section{CONCLUSÃO}

Os achados do estudo apontaram uma predileção da bronquiectasia em pacientes do sexo feminino, em idade economicamente ativa. A avaliação do SF-36 apontou uma redução da qualidade de vida em pacientes com bronquiectasia nos seguintes domínios: aspectos emocionais, aspectos físicos, estado geral de saúde e capacidade funcional.

\section{REFERÊNCIAS}

1. Guimarães FS, Moço VJR, Menezes SLS, Dias CM, Salles REB, Lopes AJ. Efeitos da ELTGOL e do Flutter® nos 
volumes pulmonares dinâmicos e estáticos e na remoção de secreção de pacientes com bronquiectasia. Rev Bras Fisioter. 2012;16(2):108-13.

2. Dalcin PTR, Perin C, Barreto SSM. Diagnóstico e tratamento das bronquiectasias: uma atualização. Rev HCPA. 2007;27(1):5160 .

3. Oliveira-Campos M, Rodrigues-Neto JF, Silveira MF, Neves DMR, Vilhena JM. The impact of risk factors of noncommunicable chronic diseases on quality of life. Ciênc Saúde Colet. 2013;18(3):873-82.

4. Silva DM. Guerreiro V, Souza SS, Francioni FF, Meirelles BHS. Qualidade de vida na perspectiva de pessoas com problemas respiratórios crônicos: a contribuição de um grupo de convivência. Rev Latinoam Enferm. 2005;13(1):7-14.

5. Conde MB. As doenças respiratórias e a atenção primária à saúde. Rev Educ Saúde. 2015; 3(2):58-63.

6. Laguardia J, Campos MR, Travassos C, Najar AL, Anjos LA, Vasconcelos MM. Dados normativos brasileiros do questionário Short Form-36 versão 2. Rev Bras Epidemiol. 2013;16(4):889-97.

7. Pimenta FAP, Simil FF, Tôrres HOG, Amaral CFS, Rezende CF, Coelho TO, et al. Avaliação da Qualidade de Vida de Aposentados com a utilização do Questionário SF-36. Rev Assoc Med Bras. 2008;54(1):55-60.

8. Lindoso VRP, Valois VM, Florenir GSP, Corrêa RGCF, Costa MRSR. Aplicação do questionário de Saint George na avaliação da qualidade de vida em pacientes com Bronquiectasia. Rev Pesq Saúde. 2013;14(1):36-9.

9. Gomes A Neto, Medeiros ML, Gifoni JMM. Bronquiectasia localizada e multissegmentar: perfil clínico-epidemiológico e resultado do tratamento cirúrgico em 67 casos. J Pneumol. 2001;27(1):1-6.

10. Cardoso AP, Polisseni N, Loivos LPP. Bronquiectasia, uma doença órfã? Pulmão. 2014;23(3):3-7.

11. Medeiros SF, Maitelli A, Nince APB. Efeitos da terapia hormonal na menopausa sobre o sistema imune. Rev Bras Ginecol Obstet. 2007;29(11):593-601.

12. Abrantes FCB, Paes FGS, Oliveira CA, Bernardes LCR, Rocha MR Júnior, Costa MVD, et al. Prevalência de bronquiectasia em pacientes do programa de assistência ao paciente asmático. Rev Pesq Saúde. 2009; 10(1):23-27.

13. Bogossian M, Santoro IL, Jamnik S, Romaldini H. Bronquiectasias: estudo de 314 casos tuberculose x não-tuberculose. J Pneumol. 1998;24(1):11-6.

14. Instituto Brasileiro de Geografia e Estatística. Síntese de indicadores sociais: uma análise das condições de vida da população brasileira: 2015. Rio de Janeiro: IBGE; 2015.

15. Barros MBA, César CLG, Carandina L, Torre GD. Desigualdades sociais na prevalência de doenças crônicas no Brasil, PNAD-2003. Ciênc Saúde Colet. 2006;11(4):911-26.

16. Faria NS Júnior, Bigatão AM, Santos SR, Leitão FSS Filho, Jardim JR, Oliveira LVF. Clinical significance in non-cystic fibrosis bronchiectasis followed in a real practice. Fisioter Mov. 2013;26(4):895-904.

17. King P, Holdsworth S, Freezer N, Holmes P. Bronchiectasis. Intern Med J. 2006;36(11):729-37.

18. Kolbe J, Wells AU. Bronchiectasis: a neglected cause of respiratory morbidity and mortality. Respirology. 1996;1(4):221-5.

19. Feltrim MI, Borges ACS, Cardoso CAL. Qualidade de vida em pacientes portadores de Pneumopatias avançadas em lista de espera de transplante pulmonar. Rev Bras Fisioter. 2006;10(10):90-9.

20. Gava M, Picanço A. Fisioterapia Pneumológica. Barueri: Manole; 2007.

21. Esteves M, Viana RA. Avaliação da qualidade de vida relacionada com a saúde na doença pulmonar obstrutiva crônica. Rev Fac Ciênc Saúde. 2010;(7):466-75.

22. Faria NS Júnior, Pasqual RM, Apostólico N, Hirata RP, Aguiar IC, Vicente R, et al. Características clínicas de pacientes portadores de bronquiectasias acompanhados de um ambulatório especializado de Pneumologia. ConScientiae Saúde. 2011;10(2):299-304.

23. Ramos-Cerqueira ATA, Crepaldi AL. Qualidade de vida em doenças pulmonares crônicas: aspectos conceituais e metodológicos. J Pneumol. 2000;26(4):207-13.

24. Branco BPC, Raso TCM, Vasconcelos TC, Araújo FV. Bronquiectasia: uma abordagem de conjunto. Rev Bras Ciênc Saúde. 2010;14(3):77-82.

25. Leal RKR. Avaliação inicial dos pacientes com bronquiectasia e aspectos do acompanhamento clínico-investigação etiológica e avaliação de qualidade. Pulmão. 2014;23(3):8-12. 
Endereço do primeiro autor:

Theila Oliveira Simas

Faculdade Santa Terezinha - CEST

Av. Casemiro, 12

Bairro: Anil

CEP: 65045-180 - São Luís - MA - Brasil

E-mail: theilasimas@hotmail.com

Endereço para correspondência:

Maysa Ferraz Reis Barroso

Hospital Universitário da Universidade Federal do Maranhão - HU/UFMA

Rua Barão de Itapari, 227

Bairro: Centro

CEP: 65020-070 - São Luís - MA - Brasil

E-mail: maysabarroso@hotmail.com 